\title{
As currently formulated, ISCN FISH nomenclature make it not practical for use in clinical test reports or cytogenetic databases
}

James T. Mascarello, $P h D^{1}$, Linda D. Cooley, $M D^{2}$, Keri Davison, $B S^{3}$, Gordon W. Dewald, $P h D^{4}$, Arthur R. Brothman, PhD , Marille Herrman, $M D, P h D^{6}$, Jonathan P. Park, PhD ${ }^{7}$, Diane L. Persons, $M D^{8}$, Kathleen W. Rao, PhD ${ }^{9}$, Nancy R. Schneider, $M D, P h D^{10}$, and Gail H. Vance, $M D^{11}$, for the Cytogenetics Resource Committee of the College of the American Pathologists and American College of Medical Genetics

\begin{abstract}
Purpose: To assess the extent and the sources of variation in ISCN nomenclature used by participants in CAP/ACMG surveys dealing with fluorescence in situ hybridization (FISH). Methods: Over 1600 nomenclature strings from 15 challenges in seven surveys were evaluated for the contributions of diagnostic errors, syntax errors, methodological differences, and technical factors not foreseen by ISCN 1995. Results: Although diagnostic errors were uncommon, syntax errors were numerous, approaching 50\% of the responses for several challenges. Their frequency varied with the complexity of the nomenclature required to describe a test condition. Variation attributable to probe selection and band designation correlated with the number of probes available for addressing the diagnostic issue at hand. In the most dramatic example of this effect, a survey simulating diagnosis of trisomy 21 in uncultured amniocytes, there were 66 participants (of 99) who used the same general form for their nomenclature, but only 8 of the 66 had exactly the same nomenclature string. Participants used proprietary names, created their own nomenclature, or ignored the true complexity of probe systems when trying to describe conditions not foreseen by ISCN 1995. Conclusion: The use of current ISCN FISH nomenclature resulted in survey participants describing unique biological conditions in a multitude of different ways. In addition to making the nomenclature unsuitable for proficiency test purposes, this heterogeneity makes it impractical for clinical test reporting and for cytogenetic database management. Because methodological information contributes a large amount of variability, adds complexity, and increases opportunities for syntax errors, a system that excludes such information would be more effective. Genet Med 2003:5(5):370-377.
\end{abstract}

Key Words: FISH, ISCN nomenclature, DNA probe, microdeletion, neoplasia

Scientific communication is often facilitated by specialized nomenclature with precisely defined terms and syntax conventions that minimize complexity and add precision to the communication process. Beginning with the ad hoc committee of

\footnotetext{
From ${ }^{I}$ Genzyme Genetics, Santa Fe, New Mexico, ${ }^{2}$ Children's Mercy Hospital, Kansas City, Missouri, ${ }^{3}$ The College of American Pathologists, Chicago, Ill, ${ }^{4}$ Mayo Clinic, Rochester, Minnesota, ${ }^{5}$ University of Utah Medical Center, Salt Lake City, Utah, ${ }^{6}$ Armed Forces Institute of Pathology, Washington, DC, ${ }^{7}$ Dartmouth-Hitchcock Medical Center, Lebanon, New Hampshire, ${ }^{8}$ University of Kansas Medical Center, Kansas City, Kansas, ${ }^{9}$ University of North Carolina, Chapel Hill, North Carolina, ${ }^{10}$ University of Texas Southwestern Medical Center, Dallas, Texas, and ${ }^{11}$ Indiana University Medical Center, Indianapolis, Indiana.

Each author is or was a member or consultant of the CAP/ACMG Cytogenetics Resource Committee. However, although the CAP/ACMG Cytogenetics Resource Committee functions under the joint sponsorship of the college of American Pathologists and the American College of Medical Genetics, this publication does not represent positions formally approved by either organization.

J.T. Mascarello, Genzyme Genetics, 2000 Vivigen Way, Santa Fe, NM 87505.

Received: March 18, 2003

Accepted: June 25, 2003
}

DOI: 10.1097/01.GIM.0000086479.80559.EA the Denver Conference and continuing, more recently, with the formalized and elected International Standing Committee for Cytogenetic Nomenclature, cytogeneticists have developed a system of definitions and conventions for describing the human karyotype. ${ }^{1,2}$ Now known as ISCN (for International System for Cytogenetic Nomenclature), the formula-like system has proven flexible enough to describe most observations made with conventional cytogenetic methods. Moreover, the system's relatively simple structure fosters correct usage and readily lends itself to the text-filtering capabilities of modern database software.

The purpose of this investigation was to determine whether the ISCN $1995^{3}$ additions dealing with fluorescence in situ hybridization (FISH) are similarly effective. The sources of data are seven FISH proficiency surveys offered jointly by the College of American Pathologists (CAP) and the American College of Medical Genetics (ACMG) between 1997 and 2001. With multiple challenges per survey and more than 100 participating laboratories, these data unquestionably represent the largest systematically collected sampling of FISH nomencla- 
ture usage. The often extreme variation in nomenclature was evaluated for the relative contributions of diagnostic error, technical variables, conditions not anticipated by ISCN 1995, and because they reflect the complexity of the system, errors in syntax.

\section{METHODS}

The FISH proficiency challenges reviewed in this study include surveys offered between 1997 and 2001. The utilization, limitations, and laboratory performance in these surveys are described elsewhere. ${ }^{4}$ This study analyzes nomenclature responses from all of the surveys except for the 2000 survey dealing with HER2/NEU gene amplification in breast cancer and the 1998 survey dealing with interphase cells from patients with chronic myeloid leukemia (CML). Nomenclature was not collected in the HER2/NEU survey. In the CML survey, some nomenclature strings exceeded the character limit of the data field in the CAP database and could not be evaluated. The remaining 7 surveys included 15 nomenclature challenges and generated 1684 responses in which participants provided the requested nomenclature.

The FISH surveys can be grouped into three categories: detection of microdeletions or microduplications in metaphase cells (1997 CYF, 1998 CYG, and 2000 CYG); detection of cells with neoplasia-related translocations in interphase cell populations (1999 CYF and 2000 CYF); and enumeration of chromosomes in interphase cells (1999 CYG and 2001 CYF). Included in the microdeletion syndromes were Williams syndrome, Prader-Willi syndrome, and velocardiofacial (VCF) syndrome. Acute promyelocytic leukemia (APL) and acute lymphoblastic leukemia (ALL) were subjects for the cancer translocation surveys, whereas prenatal diagnosis of aneuploidy and evaluation of $\mathrm{X} / \mathrm{Y}$ chimerism were subjects for the enumeration surveys. Mixtures of leukemic cells used in the APL and ALL surveys were produced by artificially mixing cell suspensions with $100 \%$ normal or neoplastic cells.

Participants were instructed to use the same DNA probes, hybridization protocols, and scoring criteria that they would use for comparable clinical evaluations. For grading purposes, participants entered a code corresponding to the multiplechoice diagnostic conclusion that best described their test results. Although it was not graded, participants were also asked to provide the ISCN 1995 nomenclature that described their results for all surveys except the 2001 survey modeling X/Y chimerism after bone marrow transplantation. In this survey, participants were told to provide nomenclature that described a hypothetical case in which they had used their own probes to detect 150 female cells and 50 male cells in a male patient who had been transplanted with cells from a female donor.

Except for the X/Y chimerism challenge and the microduplication of chromosome 15 challenge, all responses were evaluated for diagnostic correctness and for agreement between the nomenclature and the coded diagnostic conclusion. As noted above, the $\mathrm{X} / \mathrm{Y}$ chimerism nomenclature was solicited to describe a hypothetical case. Limitations in the test materials for the microduplication challenge prevented most participants from reaching the correct diagnostic conclusion.

Measuring how often survey participants misused or misunderstood ISCN 1995 guidelines was complicated by the fact that these guidelines are interpreted differently by different individuals and by the lack of guidelines for some of the situations confronted by survey participants. For the sake of consistency, syntax was scored according to the criteria used in CAP/ ACMG surveys for conventional cytogenetics but liberal allowances were made for uncertainty and/or limitations of the current guidelines. These criteria were as follows.

Missing or inappropriate use of punctuation, spaces, or parentheses; incorrect usage of "ish" or "nuc ish"; listing of nonexistent bands; and use of invalid locus names were considered syntax errors. Incorrect designation of probe status (e.g., "x1" instead of "-" for a microdeletion), incorrect order of chromosomes, anomalies, or clones; and listing a single band when designating a deletion were also considered incorrect syntax. ISCN 1995 uses a (single) band designation (e.g., 22q11.2 for the VCF syndrome critical region) to identify the probe target band when no deletion or duplication is present, but some probe manufacturers give a range of bands as the target for probes (e.g., 21q22.12-q22.2 for Vysis LSI 21). When the latter situation did (or might) apply, band ranges were considered correct. Otherwise, band ranges were considered incorrect. In the marrow transplantation survey, either cell line order (i.e., donor/recipient or recipient/donor) was considered correct.

In the context of the very large number of different nomenclature strings used by participants, recognizing patterns of nomenclature usage was difficult. To facilitate this process and to measure the contribution of probe and target band designations to the overall variation, the text handling functions of MS Access (Microsoft, Redmond, Washington) were used to replace syntactically correct probe designations and target band designations with generic designations. These functions were also used to remove bracketed numbers indicating the number of cells observed and, for the challenges modeling prenatal detection of aneuploidy in uncultured cells, to remove any portion of the response not related to evaluation of chromosomes 13,18 , and 21 . One product of this homogenization process is the "all similar strings" column in Table 3.

\section{RESULTS}

The most common nomenclature response in the challenges reviewed for this study ranged from representing $75 \%$ of the total for one of the VCF syndrome challenges down to $8 \%$ of the total for one of the prenatal diagnosis challenges. Diagnostic errors, syntax errors, and differing methods for describing situations not anticipated in ISCN 1995 contributed to the nomenclature variation in all of the surveys. For those survey challenges based on mixtures of cells, responses also varied with respect to whether and how the mixtures were described.

Diagnostic errors were rare (Table 1), exceeding a few percent only for those challenges in which the abnormal cell type constituted a minority of the cells in the sample. For the 21 
instances in which participants reached the wrong diagnostic conclusion, 15 used the correct nomenclature to describe that incorrect diagnosis. For the other six, the syntax was incorrect but the nomenclature was, nevertheless, interpretable as describing the incorrect diagnosis. There were 13 instances, distributed fairly evenly over the six surveys, in which participants made the correct diagnosis and yet used nomenclature that clearly described a different condition. Although the diagnostic conclusion (a mixture of XX and XY cells) was provided in the $\mathrm{X} / \mathrm{Y}$ chimerism survey materials, one participant wrote nomenclature that described a mixture of XX and XXY cells.

Ranging from $11 \%$ to $49 \%$ of all responses, syntax errors (errors in nomenclature usage) were far more common than diagnostic errors (Table 1). Syntax errors that are common in the CY (G-banding) proficiency surveys were also common in the FISH surveys. These included nonexistent band listings, incorrect order of cell lines, and missing, additional, or inap- propriate use of punctuation. FISH nomenclature includes more opportunities to use spaces (e.g., "ish $\operatorname{del}(22) \ldots$.." or "ABL con BCR"), so omitted spaces contributed significantly to the number of syntax errors. For nomenclature specifically formulated to describe FISH observations, common syntax errors included the following: use of " $\mathrm{x} 1$ " instead of "-" to describe a deletion; use of "+" instead of "x2" when no deletion was present; use of "-" to describe the absence of a Y probe signal in female cells; listing a single band to describe a deletion; listing 2 bands when no deletion was present; repetitive use of "ish" or "nuc ish"; and use of proprietary (manufacturers') names for probes.

Syntax errors tended to be less common in the surveys relating to microdeletions in metaphase cells and more common in surveys dealing with interphase cells from neoplasia. Confusion over the order of cell lines and how to describe juxtaposed probe signals accounts for most of the difference. Surprisingly,

Table 1

Diagnosis and syntax errors

\begin{tabular}{|c|c|c|c|c|c|c|c|c|c|c|c|}
\hline & \multirow[b]{3}{*}{$N$} & \multicolumn{6}{|c|}{ Syntax errors ${ }^{a}$} & \multicolumn{4}{|c|}{ Diagnosis errors $^{b}$} \\
\hline & & \multicolumn{2}{|c|}{$\begin{array}{l}\text { Correct Dx } \\
\text { and syntax }\end{array}$} & \multirow{2}{*}{$\frac{\text { Correct Dx }}{n}$} & \multirow{2}{*}{$\frac{\text { Incorrect Dx }}{n}$} & \multicolumn{2}{|c|}{ Total } & \multirow{2}{*}{$\frac{\text { Correct syntax }}{n}$} & \multirow{2}{*}{$\frac{\text { Incorrect syntax }}{n}$} & \multicolumn{2}{|c|}{ Total } \\
\hline & & $n$ & $\%$ & & & $n$ & $\%$ & & & $n$ & $\%$ \\
\hline \multicolumn{12}{|c|}{ VCFS, 1997 CYF } \\
\hline VCF -1 & 117 & 101 & $86.3 \%$ & 16 & 0 & 16 & $13.7 \%$ & 0 & 0 & 0 & $0.0 \%$ \\
\hline $\mathrm{VCF}-2$ & 117 & 94 & $80.3 \%$ & 18 & 3 & 21 & $17.9 \%$ & 1 & 1 & 2 & $1.7 \%$ \\
\hline \multicolumn{12}{|c|}{ Williams S, 1998 CYG } \\
\hline WS -1 & 109 & 90 & $82.6 \%$ & 19 & 0 & 19 & $17.4 \%$ & 0 & 0 & 0 & $0.0 \%$ \\
\hline WS -2 & 109 & 95 & $87.2 \%$ & 12 & 0 & 12 & $11.0 \%$ & 2 & 0 & 2 & $1.8 \%$ \\
\hline \multicolumn{12}{|c|}{ Acute Promy Leuk, 1999 CYF } \\
\hline $\mathrm{APL}-1$ & 106 & 78 & $73.6 \%$ & 27 & 0 & 27 & $25.5 \%$ & 1 & 0 & 1 & $0.9 \%$ \\
\hline $\mathrm{APL}-2$ & 107 & 91 & $85.0 \%$ & 12 & 1 & 13 & $12.1 \%$ & 2 & 1 & 3 & $2.8 \%$ \\
\hline $\mathrm{APL}-3$ & 107 & 59 & $55.1 \%$ & 41 & 3 & 44 & $41.1 \%$ & 4 & 0 & 4 & $3.7 \%$ \\
\hline \multicolumn{12}{|c|}{ Prenatal Dx, 1999 CYG } \\
\hline PND - 1 & 99 & 67 & $67.7 \%$ & 30 & 2 & 32 & $32.3 \%$ & 0 & 0 & 0 & $0.0 \%$ \\
\hline PND - 2 & 103 & 64 & $62.1 \%$ & 35 & 2 & 37 & $35.9 \%$ & 0 & 2 & 2 & $1.9 \%$ \\
\hline \multicolumn{12}{|c|}{ Acute Lym Leuk, 2000 CYF } \\
\hline $\mathrm{ALL}-1$ & 100 & 57 & $57.0 \%$ & 42 & 1 & 43 & $43.0 \%$ & 0 & 0 & 0 & $0.0 \%$ \\
\hline $\mathrm{ALL}-2$ & 101 & 82 & $81.2 \%$ & 19 & 0 & 19 & $18.8 \%$ & 0 & 1 & 1 & $1.0 \%$ \\
\hline $\mathrm{ALL}-3$ & 101 & 55 & $54.5 \%$ & 38 & 1 & 39 & $38.6 \%$ & 5 & 1 & 6 & $5.9 \%$ \\
\hline \multicolumn{12}{|c|}{ Prader-Willi S, 2000 CYG } \\
\hline PWS - 1 & 148 & 113 & $76.4 \%$ & 35 & 0 & 35 & $23.6 \%$ & 0 & 0 & 0 & $0.0 \%$ \\
\hline PWS - 2 & 148 & $\cdots$ & $\cdots$ & 54 & $\cdots$ & 54 & $36.5 \%$ & $\cdots$ & $\cdots$ & . & $\cdots$ \\
\hline \multicolumn{12}{|c|}{ X/Y Chimerism, 2001 CYF } \\
\hline $\mathrm{X} / \mathrm{Y}$ & 112 & 57 & $50.9 \%$ & 54 & 1 & 55 & $49.1 \%$ & $\ldots$ & $\ldots$ & $\ldots$ & $\ldots$ \\
\hline
\end{tabular}

a"Syntax Errors" refers to participants who provided the correct diagnosis code for the challenge but used incorrect syntax to describe the diagnosis. This section is further subdivided into responses from which the correct diagnosis might be inferred and responses that clearly describe the wrong diagnosis.

b"Diagnosis errors" refers to responses with incorrect diagnosis codes. These are subdivided into responses accompanied by correct nomenclature (for the wrong diagnosis) and those accompanied by incorrect nomenclature. In no case, was nomenclature for the correct diagnosis submitted with an incorrect diagnosis code. 
the challenge dealing with the simulated bone marrow transplant generated the highest rate of syntax errors (49\%). Errors in this challenge included virtually all of those listed above with no particular one accounting for a disproportionately large fraction.

Heterogeneity in nomenclature was also a consequence of variation in probe designation and variation in the band(s) to which probe signals were assigned. Note that for purposes of this presentation, the term, probe name/designation, is used instead of locus name/designation. ISCN recommends using Genome Database (GDB) names whenever available but, as will become apparent, GDB locus names cannot completely describe the complexity of many probe systems in current use. Table 2 summarizes the usage of probe names. In only the 1999 acute promyelocytic leukemia (APL) survey was there complete homogeneity for probe designation. For other surveys, alternative names for the probes and/or alternative probes added to the complexity of the nomenclatures. Noteworthy, with respect to the latter, was the 1999 simulation of chromo- some enumeration in uncultured amniocytes. In the first challenge, cells from a fetus with trisomy 21 , there were 11 different designations for the chromosome 13 probe, 4 different designations for the chromosome 18 probe, and 29 different designations for the chromosome 21 probe.

The impact of probe and band designation on nomenclature variation is examined in Table 3 . The table compares the frequency of the single most commonly used nomenclature string to the frequency of all similar syntactically correct strings. The latter group includes the most common string as well as all other strings that differ only with respect to band and/or probe designation. The form expected for these strings is represented with generic labels in Table 3. The difference between the frequency of the most common string and the frequency of all strings like it is a rough measure of the contribution that band and probe designation make to the overall variation in nomenclature. This difference ranges from a low of $5 \%$ in the second VCF syndrome metaphase challenge to a high of $59 \%$ in one of the prenatal diagnosis interphase challenges. Variation in band

Table 2

Variation in Probe Designation

$N$

VCFS, 1997 CYF

$\mathrm{VCF}-1$

$\mathrm{VCF}-2$

Acute Promy Leuk, 1999

CYF

APL -1

APL -2

107

APL -3

Prenatal Dx, 1999 CYG

PND -1

Acute Lymph Leuk, 2000

CYF

ALL - 1

100

ALL -2

101

ALL - 3

Prader-Willi S, 2000 CYG

$$
\text { PWS }-1
$$

PWS - 2

X/Y Chimerism, 2001 CYF

$\mathrm{X} / \mathrm{Y}$
Probe designations (includes different names for the same gene) ${ }^{a}$

D22S75(108); Tuple1(5); Tuple1 mix(1); D22S57(1); D22S53/D22S609(1); LSI(1)

D22S75(108); Tuple1(5); Tuple1 mix(1); D22S75(1); D22S53/D22S609(1); LSI(1)

D7S427(2); D7S486(2); ELN(94); ELN mix(6); WSCR(5)

D7S427(3); D7S486(2); ELN(93); ELN mix(6); WSCR(5)

PML(106); RARA(106)

PML(107); RARA(107)

PML(107); RARA(107)

11 different designations for chrom 13 probes; 4 different for 18; 29 different for 21

9 different designations for chrom 13 probes; 4 different for 18; 22 different for 21

TEL(96); TEL-ETV6(3); ETV6(1); AML1(96); AML(4)

TEL(97); TEL-ETV6(3); ETV6(1); AML1(94); $\operatorname{AML(7)~}$

TEL(97); TEL-ETV6(3); ETV6(1); AML1(94); $\operatorname{AML(7)~}$

SNRPN(74); SNRPN mix(71); D15S10(2); GABARB3(1)

SNRPN(76); SNRPN mix(68); D15S10(3); GABARB3(1)

${ }^{a}$ Except for the prenatal diagnosis survey, the number of times a probe designation was used is given in parentheses after that designation. The term "mix" is used whenever a primary probe was used in combination with a mixture of other probes. 
Table 3

Impact of variation in probe and band designation

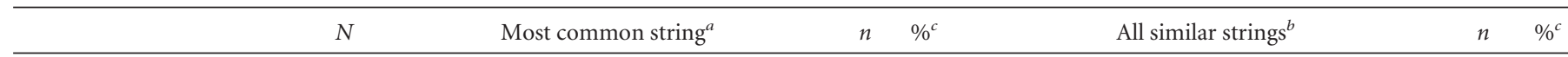

VCFS, 1997 CYF

$\mathrm{VCF}-1$

VCF -2

117 ish del(22)(q11.2q11.2)(D22S75-)

117 ish 22q11.2(D22S75x2)

Williams S, 1998 CYG
WS -1
109 ish $\operatorname{del}(7)(\mathrm{q} 11.23 \mathrm{q} 11.23)(\mathrm{ELN}-)$
WS -2
109 ish 7q11.23(ELNx2)

Acute Promy Leuk, 1999 CYF

$$
\begin{aligned}
& \text { APL }-1 \\
& \text { APL }-2 \\
& \text { APL - } 3
\end{aligned}
$$

Prenatal Dx, 1999 CYG

$$
\text { PND }-1
$$

PND -2

\author{
106 nuc ish 15q22(PMLx2), 17q21.1(RARAx2) \\ (PML con RARAxl) \\ 107 nuc ish 15q22(PMLx2), 17q21.1(RARAx2) \\ 107 nuc ish 15q22(PMLx2), 17q21.1(RARAx2) \\ (PML con RARAx1)
}
99 nuc ish $13 \mathrm{q} 14(\mathrm{RB} 1 \mathrm{x} 2)$,
18cen(D18Z1x2),21q22.13-q22.2
(D21S259x3,D21S341x3,D21S342x3)
103 nuc ish $13 \mathrm{q} 14(\mathrm{RB} 1 \times 3)$,
18cen(D18Z1x3),21q22.13-q22.2
(D21S259x3,D21S341x3,D21S342x3)

$9682 \%$

$90 \quad 77 \%$

$84 \quad 72 \%$ ish chromband(probex 2 )
$83 \quad 76 \%$

$88 \quad 81 \%$

$77 \quad 71 \%$ ish chromband(probex 2 )

$2120 \%$ nuc ish chromband(probex2), chromband(probex2)(probe con probex 1 )

$2725 \%$ nuc ish chromband(probex2), chromband(probex2)

$109 \%$ nuc ish chromband(probex2), chromband(probex2)(probe con probex1)

$69 \quad 65 \%$

$88 \quad 82 \%$

$51 \quad 48 \%$

$8 \% \quad$ nuc ish 13band(probex2),
18band(probex2),21band(probex3)

$66 \quad 67 \%$

$11 \quad 11 \%$ nuc ish 13band(probex3), 18band(probex3),21band(probex3)

Acute Lym Leuk, 2000 CYF

$$
\begin{aligned}
& \text { ALL - } 1 \\
& \text { ALL - } 2 \\
& \text { ALL - } 3
\end{aligned}
$$

100 nuc ish 12p13(TELx2),21q22(AML1x2)(TEL con AML1x1)

101 nuc ish 12p13(TELx2),21q22(AML1x2)

101 nuc ish 12p13(TELx2),21q22(AML1x2) (TEL con AML1x1)

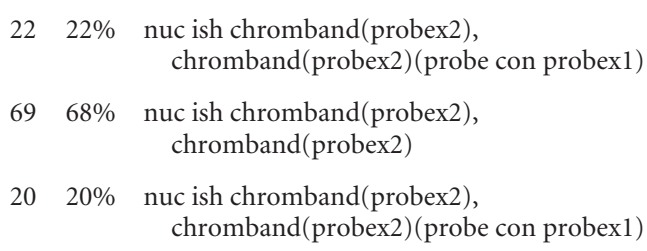

Prader-Willi S, 2000 CYG

$$
\begin{aligned}
& \text { PWS - } 1 \\
& \text { PWS - } 2
\end{aligned}
$$$$
148 \text { ish del(15)(q11.2q11.2)(SNRPN-) }
$$

148 ish 15q11.2(SNRPNx2)

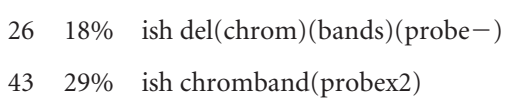

$106 \quad 72 \%$

$82 \quad 55 \%$

X/Y Chimerism, 2001 CYF $\mathrm{X} / \mathrm{Y}$

112 nuc ish Xcen(DXZ1x2)[150]/Xcen(DXZ1x1), Ycen(DYZ3x1)[50]

$1614 \%$ nuc ish Xband(probex2)[150]/Xband(probex1), Yband(probex1)[50]

${ }^{a}$ The "Most common string" section of the table illustrates the form as well as the number and percentage of responses with the most common nomenclature string. Such responses were identical in every respect.

${ }^{b}$ The "All Similar Strings" section refers to all syntactically correct responses resembling (and including) the most common string. These strings differed from each other only with respect to probe and/or target band designation. The general form of these responses is illustrated by strings in which generic labels have been substituted for the specific probe and band designations.

"The difference between the frequencies (\%) for "all similar" and "most common" strings is a rough measure of the impact of band and probe designation on the variation in nomenclature and ranges from a low of $5 \%$ to a high of $59 \%$.

designation is both a consequence of different probes having different target bands and a consequence of participants using different band levels to describe the target band. The latter led to considerable nomenclature variation in the APL challenges (in which all participants used exactly the same probes).

In the two leukemia surveys and in the survey dealing with chimerism subsequent to bone marrow transplantation, participants could have used nomenclature to describe mixtures of cells in any or all of the challenges. Table 4 compares test conditions in these surveys to the number of participants who used nomenclature indicating the presence of more than one cell line. In most cases, a mixture of cells was indicated simply by the listing of two cell lines with a "/" separator. The triplet "mos" was used only once, in a response from the APL challenge with $19 \%$ neoplastic cells. The triplet "chi" was used by three participants in the $\mathrm{X} / \mathrm{Y}$ chimerism survey. Even though one ALL challenge included no neoplastic cells, there were still three participants who described multiple cell lines. Similarly, 
Table 4

Reporting mixed cell types

\begin{tabular}{cc}
\hline $\begin{array}{c}\text { Frequency of } \\
\text { abnormal cells, } \\
\%\end{array}$ & $\begin{array}{c}\text { Responses } \\
\text { indicating two } \\
\text { cell lines, } \%^{a}\end{array}$ \\
\hline
\end{tabular}

Acute Promy Leuk, 1999 CYF

$\begin{array}{lrr}\text { APL }-1 & 92 \% & 27 \% \\ \text { APL }-2 & 0 \% & 22 \% \\ \text { APL }-3 & 19 \% & 67 \%\end{array}$

Acute Lym Leuk, 2000 CYF

$\begin{array}{lrr}\text { ALL }-1 & 50 \% & 50 \% \\ \text { ALL }-2 & 0 \% & 3 \% \\ \text { ALL }-3 & 20 \% & 48 \%\end{array}$

X/Y Chimerism, 2001 CYF

$\mathrm{X} / \mathrm{Y}$

$25 \%$

$100 \%$

${ }^{a}$ For each of the challenges in which a mixture of cells was (or might have been) present, the frequency of leukemic cells, or in the case of the X/Y chimerism challenge the frequency of recipient cells, is compared to the proportion of participants who used nomenclature describing multiple cell lines. Note that many participants omitted description of the second cell line when one was clearly present and that some participants described a second cell line when none was present.

in the APL challenges that included $92 \%$ and $0 \%$ neoplastic cells, $27 \%$ and $22 \%$, respectively, of the participants wrote nomenclature that described multiple cell lines. In most cases, the number of cells listed as having the less common signal pattern was very low. Thus, for the APL challenge with $92 \%$ neoplastic cells these designations could be an accurate representation of the minority cell population. For the challenge with no neoplastic cells, these designations could be a consequence of random juxtaposition of probe signals producing an artifactual fusion type signal pattern.

The leukemia surveys also revealed a potential source of confusion with nomenclature strings that used the triplet "con." In the ALL survey, two participants used the form “...,12p13(TELx2)(TEL con AML1x1),..." to describe the interphase nuclei of cells with the $t(12 ; 21)$ translocation. This nomenclature places the probe fusion signal on the derivative 12. The commercially available probe set used by most, if not all, participants produces a fusion signal over the derivative 21, so it is likely that this nomenclature was used in error. Nevertheless, the fact that there could be a TEL/AML1 probe set that really does produce a fusion signal over the derivative 12 makes it necessary for one to take this designation at face value. Because single fusion FISH strategies give little or no information about the disposition of the reciprocal chromosome fragments, “...,12p13(TELx2)(TEL con AML1x1),21q22(AML1x2)..." and “...,12p13(TELx2),21q22(AML1x2)(TEL con AML1x1)" are designations that could be associated with different biological conditions.

Technological factors not anticipated by ISCN 1995 constitute the last source of variation in the nomenclature submitted by survey participants. The difficulties arising from these fac- tors were also not anticipated when the surveys were designed, so there is no way to measure their full impact. Nevertheless, the following uncertainties were evident from the responses submitted and from the letters that some participants submitted with their test results.

1. Some commercial "probes" are actually mixes of sequences from all or part of several adjacent loci. Some participants attempted to document such mixes by listing probe names with either slashes or commas as separators. The difficulty with these mixes is that the user cannot know whether the signal seen represents one or all of the loci contained in the mix. Conversely, the absence of signal need not mean that all of the loci have been deleted.

2. Product literature sometimes describes a probe's target as a range of bands. For example, Vysis describes its LSI 21 product (a mixture of sequences from D21S259, D21S341, and $\mathrm{D} 21 \mathrm{~S} 342$ ) as mapping to $21 \mathrm{q} 22.13-\mathrm{q} 22.2$. This designation is not consistent with ISCN 1995, but might be an appropriate representation of the certainty with which some targets have been mapped.

3. The only commercially available probe system for the $\mathrm{t}(12 ; 21)$ translocation in ALL is one that is intended to produce a strong hybrid signal (TEL-AML1) as well as an extra weak AML1 signal when the translocation is present. The total number of AML1 signals in leukemic cells should be three. Despite this fact and despite the fact that the overwhelming majority of survey participants used commercial probes, over half of all of the nomenclature strings submitted for the challenges with leukemic cells described the presence of only two AML1 signals. Thus, most participants either incorrectly described the extra signal pattern or, for technical reasons, were unable to see the extra signal.

4. Low-level "mosaicism" in analyses performed on interphase cells may have its basis in artifact. Laboratories use reference ranges in an attempt to deal with this uncertainty but ISCN has no construct for incorporating reference range information.

\section{DISCUSSION}

In the CAP/ACMG surveys dealing with traditional (CY) cytogenetic banding methods, survey participants use ISCN nomenclature to describe their test results. Although some challenges yield relatively complex nomenclature strings, syntax errors are infrequent enough that the overwhelming majority of challenges meet the minimum $80 \%$ consensus criterion required for grading nomenclature. Moreover, attempts to replace ISCN nomenclature with arbitrary codes corresponding to chromosomes and abnormalities (in early CAP/ ACMG surveys) led to an increase in clerical errors and to participant complaints regarding this departure from a system that was familiar to most cytogeneticists. Despite the favorable experience with the CY surveys, the complex variation of nomenclature submitted in pilot FISH surveys ${ }^{5}$ caused the committee responsible for the surveys to relegate nomenclature to "supplementary" (not graded) question status when formal grading of FISH proficiency surveys began in 1999. 
As evident from the results described in this study, this cautious approach was warranted. Had the grading rules for CY surveys been used, and had any valid probe and band designation been allowed, only 5 of the 14 graded challenges would have had nomenclature that met the minimum $80 \%$ consensus criterion (the second 2000 CYG challenge was not graded). Diagnostic errors were uncommon but, in some challenges, nearly half of the responses contained syntax errors. Both between and within surveys, the number of syntax errors appears to correlate with the complexity of the nomenclature required to describe the test condition (see Tables 1 and 2). For instance, in the APL survey, the challenge that included no neoplastic cells generated only $12 \%$ syntax errors. The challenge with $19 \%$ neoplastic cells generated $41 \%$. The meaning of nomenclature strings is usually not obscured by syntax errors, but interpreting such strings requires assumptions that would introduce undesirable subjectivity into the proficiency test grading process.

Structured education and practice (perhaps via proficiency testing programs) might enhance user familiarity with FISH nomenclature conventions and might reduce the frequency of syntax errors to the point where the nomenclature could be a practical medium for communicating proficiency test results. However, this is a relatively unimportant aspect of the nomenclature's intended purpose. More important is the value of such a system for communicating observations between geneticists and for cataloging cytogenetic data in a manner that readily fosters correlation with clinical observations. Ideally, such a system would be simple enough to be understood by clinicians who routinely order cytogenetic testing. The experience obtained from the CAP/ACMG FISH proficiency surveys suggests that the current system has serious limitations with regard to all of these objectives.

Some of the limitations could be remedied by expanding the nomenclature guidelines to include standards for the following:

1. Probe/locus name or designation.

2. Band level at which a probe's target should be listed.

3. Thresholds for describing low-frequency cell types in interphase nuclei populations.

4. The order of (normal) cell lines ascertained in bone marrow transplant patients.

5. Listing (or not) the status of control probes used in most diagnostic FISH testing.

6. Listing (or not) the status of $Y$ chromosome probes in XX nuclei that have been tested for sex chromosome constitution.

Dealing with the problem of probe names and their expected signal patterns would seem to be particularly daunting. Even if they target the same locus, probes prepared by different laboratories (or manufacturers) often have unique molecular constructs that lead to differences in their resulting signal patterns. As illustrated by FISH tests for cancer translocations, this problem is exacerbated when more than one probe is used. Currently, there are cancer probe sets that demonstrate the translocation with double fusion signals and probe sets that demonstrate the translocation with a single fusion signal. Single fusion probe sets can be designed such that the fusion signal appears over either of the derivative chromosomes and they can also be designed such that the translocation leaves an extra (residual) signal on one of the translocation partners. Thus, there are at least four different designs for single fusion probe sets and a total of five possible signal patterns when double fusion probe sets are included. A different nomenclature string would be required to describe each of the five signal patterns.

The practice of identifying probes by locus names is also a problem for those probes that target a region within a gene (e.g., the Cytocell panel of probes used for evaluating the deletion hot spots in the dystrophin gene) and those that consist of sequences from contiguous loci (e.g., the combination of ELN, LIMK1, and D7S613 in the Vysis product for detecting Williams syndrome deletions).

Assuming that simple probe-naming conventions could be devised, the description of some conditions demonstrable by FISH would still be complicated by our ability to use a variety of probes to accomplish the same investigation. In the challenge simulating prenatal diagnosis of a trisomy 21 fetus using uncultured amniocytes, there were 1276 possible combinations of the probe designations used in the enumeration of chromosomes 13, 18, and 21

FISH nomenclature differs from the nomenclature for banded chromosomes in two important respects. First, the latter is inherently efficient because, for the most part, only deviations from the expected normal karyotype are described. FISH nomenclature describes the result of all testing, regardless of whether the conditions observed are normal or abnormal. The second is that banding nomenclature omits the technical basis for the observations (i.e., G-, R-, or Q-banding), whereas FISH nomenclature includes it (i.e., the probes). If probe information were located elsewhere (as is the case for banding information in most clinical reports), the length and complexity of the nomenclature strings and, hence, the opportunities for syntax errors would all be significantly reduced.

With probe information removed from the nomenclature, a Williams syndrome microdeletion might be written, 46,XY.ish $\operatorname{del}(7)$ (q11.23q11.23) while a normal result for this FISH test might be written 46,XY.ish 7q11.23. Evidence of aneuploidy in uncultured amniocytes might be written nuc ish $13 \mathrm{q} 14,18 \mathrm{cen},+21 \mathrm{q} 22$. This notation describes the presence of an extra FISH signal corresponding to band 21q22. It is consistent with trisomy 21 but owing to the limitations of interphase FISH, cannot discriminate between trisomy 21 and a duplication of band 21q22. Evidence of a translocation in nuclei from a patient with chronic myeloid leukemia might be written nuc ish $\mathrm{t}(9$; 22)(q34;q11.2) or nuc ish der(22)t(9;22)(q34;q11.2) depending on whether the probe set produced a dual fusion signal or produced a single fusion signal over the derivative 22 . Table 5 provides other examples specifically relating to $\mathrm{BCR} / \mathrm{ABL}$ probe sets. Note, that by omitting technical detail, the nomencla- 
Table 5

FISH nomenclature without probe data

\begin{tabular}{|c|c|c|c|}
\hline Test condition & $\begin{array}{l}\text { FISH strategy: signal } \\
\text { pattern }^{a}\end{array}$ & $\begin{array}{l}\text { Nomenclature without probe } \\
\text { information }\end{array}$ & Comment \\
\hline Normal result & $\begin{array}{l}\text { SF, DF or ES: } 2 \text { red and } \\
2 \text { green }\end{array}$ & nuc ish $9 \mathrm{q} 34,22 \mathrm{q} 11.2$ & $\begin{array}{l}\text { The nomenclature lists the interrogated } \\
\text { bands and, because no abnormality is } \\
\text { described, the normal condition is implied. }\end{array}$ \\
\hline \multirow[t]{3}{*}{$\mathrm{t}(9 ; 22)$} & $\begin{array}{l}\text { DF: } 1 \text { red, } 1 \text { green, } 2 \\
\text { fusion }\end{array}$ & nuc ish $\mathrm{t}(9 ; 22)(\mathrm{q} 34 ; \mathrm{q} 11.2)$ & $\begin{array}{l}\text { Only the DF system can detect both } \\
\text { translocation products }\end{array}$ \\
\hline & $\begin{array}{l}\text { SF: } 1 \text { red, } 1 \text { green, } 1 \\
\text { fusion }\end{array}$ & nuc ish $\operatorname{der}(22) \mathrm{t}(9 ; 22)(\mathrm{q} 34 ; \mathrm{q} 11.2)$ & SF system detects only the der(22) \\
\hline & $\begin{array}{l}\text { ES: } 2 \text { red, } 1 \text { green, } 1 \\
\quad \text { fusion }\end{array}$ & nuc ish $\operatorname{der}(22) \mathrm{t}(9 ; 22)(\mathrm{q} 34 ; \mathrm{q} 11.2)$ & $\begin{array}{l}\text { ES system detects der }(22) \text { and extra signal } \\
\text { but gives no information about where the } \\
\text { extra signal is }\end{array}$ \\
\hline \multirow{3}{*}{$\begin{array}{l}\mathrm{t}(9 ; 22) \text { with } \\
\text { concomitant } \\
\text { deletion in the } \\
\text { der(9) }\end{array}$} & $\begin{array}{l}\text { SF: } 1 \text { red, } 1 \text { green, } 1 \\
\text { fusion }\end{array}$ & nuc ish $\operatorname{der}(22) \mathrm{t}(9 ; 22)(\mathrm{q} 34 ; \mathrm{q} 11.2)$ & SF system detects only the der(22) \\
\hline & $\begin{array}{l}\text { ES: } 1 \text { red, } 1 \text { green, } 1 \\
\quad \text { fusion }\end{array}$ & nuc ish $-9 \mathrm{q} 34, \operatorname{der}(22) \mathrm{t}(9 ; 22)(\mathrm{q} 34 ; \mathrm{q} 11.2)$ & $\begin{array}{l}\text { ES system detects derivative } 22 \text { and loss of } \\
\text { centromeric portion of } A B L\end{array}$ \\
\hline & $\begin{array}{l}\text { DF: } 1 \text { red, } 2 \text { green, } 1 \\
\text { fusion }\end{array}$ & nuc ish $-9 \mathrm{q} 34, \operatorname{der}(22) \mathrm{t}(9 ; 22)(\mathrm{q} 34 ; \mathrm{q} 11.2)$ & $\begin{array}{l}\text { DF system detects derivative } 22 \text { and loss of } \\
\text { centromeric portion of ABL }\end{array}$ \\
\hline
\end{tabular}

${ }^{a}$ Demonstration of three test conditions by single fusion (SF), double fusion (DF), or extra signal (ES) FISH strategies.

${ }^{b} \mathrm{~A}$ possible form of nuc ish nomenclature that does not include probe information. The nomenclature system assumes the following: that anything not specified has not been tested; normal result can be indicated by listing only the chromosome(s) and band(s) interrogated by the probes; the nomenclature reports only that which can be inferred from the signal pattern and; "-" and "+" are used to indicate missing or extra copies of the interrogated band.

ture system could also be extended to include results from DNAbased testing (e.g., 46,XY.pcr t(12;21)(p13;q22)).

The system of terms and syntax guidelines incorporated into the 1995 version of ISCN was proposed by the International Standing Committee on Human Cytogenetic Nomenclature in 1994. The system was effective at describing FISH observations likely to be made with the technologies then available, but in the last nine years, new technologies and new applications have severely taxed its capabilities. A system that fosters precise and succinct descriptions of FISH observations has obvious value for reporting test results and for facilitating clinical correlations. There is, therefore, an urgent need for nomenclature guidelines that deal with some of the issues and sources of variation identified in this report.

\section{References}

1. Hamerton JL. Chromosome band nomenclature: The Paris Conference, 1971. In: Caspersson T, Zech L, editors. Chromosome identification: Technique and applications in biology and medicine. Stockholm, Sweden: Academic Press, 1973:90-97.

2. International Standing Committee on Human Cytogenetic Nomenclature; Harnden DG, Klinger HP, editors. An international system for human cytogenetic nomenclature. Basel, Switzerland: S. Karger AG; 1985.

3. International Standing Committee on Human Cytogenetic Nomenclature; Mitelman F, editor. An International System for Human Cytogenetic Nomenclature. Basel, Switzerland: S. Karger AG; 1995.

4. Mascarello JT, Brothman AR, Davison K, Dewald GW, Herrman M, McCandless $\mathrm{D}$ et al. Proficiency testing for laboratories performing fluorescence in situ hybridization with chromosome-specific DNA probes. Arch Pathol Lab Med 2002; 126:1458-1462.

5. Dewald GW, Brothman AR, Butler MG, Cooley LD, Patil SR, Saikevych IA et al. Pilot studies for proficiency testing using fluorescence in situ hybridization with chromosome-specific DNA probes: A College of American Pathologists/American College of Medical Genetics Program. Arch Pathol Lab Med 1997;121:359-367. 